(c) (1)

\title{
The inbuilt long-term unfeasibility of environmental flows when disregarding riparian vegetation requirements
}

\author{
R. Rivaes et al. \\ Correspondence to: R. Rivaes (ruirivaes@isa.ulisboa.pt)
}

The copyright of individual parts of the supplement might differ from the CC-BY 3.0 licence. 
Supplement S1: Hydraulic data and channel bed characteristics for River 2D and CASiMiR-vegetation model parameterization

Table S1. Flow curve considered in the downstream section as the outflow condition in River2D model.

\begin{tabular}{l|l}
\hline $\begin{array}{l}\text { Discharge } \\
\left(\mathrm{m}^{3} \mathrm{~s}^{-1}\right)\end{array}$ & $\begin{array}{l}\text { Water } \\
\text { surface } \\
\text { elevation } \\
(\mathrm{m})\end{array}$ \\
\hline 0.05 & 139.65 \\
0.1 & 139.67 \\
0.3 & 139.80 \\
0.5 & 139.92 \\
0.7 & 140.00 \\
1 & 140.08 \\
2 & 140.25 \\
3 & 140.39 \\
4 & 140.50 \\
\hline
\end{tabular}

Table S2. Channel roughness classification of the different substrates in the aquatic zone of the river without vegetation used in the River2D model.

\begin{tabular}{l|l}
\hline Substrate & $\begin{array}{l}\text { Effective } \\
\text { roughness } \\
\text { height, } k_{s}(\mathrm{~m})\end{array}$ \\
\hline Sand and gravel & 0.1 \\
Boulders and stones & 0.5 \\
\hline
\end{tabular}


Table S3. Channel roughness classification of the different considered riparian vegetation succession phases used in the River2D model (IP - Initial phase, PP Pioneer phase, ES - Early succession woodland phase, EF - Established forest phase and MF - Mature forest phase).

\begin{tabular}{l|l}
\hline $\begin{array}{l}\text { Succession } \\
\text { phase }\end{array}$ & $\begin{array}{l}\text { Effective } \\
\text { roughness } \\
\text { height, } k_{s}(\mathrm{~m})\end{array}$ \\
\hline $\mathrm{IP}$ & 0.4 \\
$\mathrm{PP}$ & 0.5 \\
$\mathrm{ES}$ & 1.6 \\
$\mathrm{EF}$ & 1.1 \\
$\mathrm{MF}$ & 1.4 \\
\hline
\end{tabular}


Table S4. CASiMiR-vegetation model parameterization (IP - Initial phase, PP Pioneer phase, ES - Early succession woodland phase, EF - Established forest phase and MF - Mature forest phase).

\begin{tabular}{|c|c|c|}
\hline Parameter & $\begin{array}{l}\text { Succession } \\
\text { phase }\end{array}$ & Value \\
\hline $\begin{array}{l}\text { Height to } \\
\text { water table } \\
\text { elevation }(\mathrm{m})\end{array}$ & $\begin{array}{l}\text { IP } \\
\text { PP } \\
\text { ES } \\
\text { EF } \\
\text { MF }\end{array}$ & $\begin{array}{l}<0.2 \\
0.2-0.6 \\
0.6-1.05 \\
1.05-3.4 \\
>3.4\end{array}$ \\
\hline Age (years) & $\begin{array}{l}\text { IP } \\
\text { PP } \\
\text { ES } \\
\text { EF } \\
\text { MF }\end{array}$ & $\begin{array}{l}<2 \\
2-6 \\
6-19 \\
19-26 \\
>26\end{array}$ \\
\hline $\begin{array}{l}\text { Resistance to } \\
\text { shear stress } \\
\left(\mathrm{N} \mathrm{m}^{-2}\right)\end{array}$ & $\begin{array}{l}\text { IP } \\
\text { PP } \\
\text { ES } \\
\text { EF } \\
\text { MF }\end{array}$ & $\begin{array}{l}30 \\
30 \\
50 \\
300 \\
300\end{array}$ \\
\hline
\end{tabular}




\section{Supplement S2: Summary of collected data supporting the}

ecological background for riparian vegetation and fish modeling.

Table S5. Patch characterization of succession phases (IP - Initial phase, PP - Pioneer phase, ES - Early succession woodland phase, EF - Established forest phase and MF Mature forest phase).

\begin{tabular}{|c|c|c|c|c|c|c|c|}
\hline $\begin{array}{l}\text { Succession } \\
\text { phase }\end{array}$ & $\begin{array}{l}\text { Patches } \\
\text { surveyed }\end{array}$ & $\begin{array}{l}\text { Mean } \\
\text { height to } \\
\text { mean } \\
\text { water } \\
\text { level(m) }\end{array}$ & $\begin{array}{l}\text { Mean } \\
\text { area } \\
\left(\mathrm{m}^{2}\right)\end{array}$ & $\begin{array}{l}\text { Mean } \\
\text { cover of } \\
\text { herb } \\
\text { layer } \\
(\%)\end{array}$ & $\begin{array}{l}\text { Mean } \\
\text { cover of } \\
\text { shrub } \\
\text { layer } \\
(\%)\end{array}$ & $\begin{array}{l}\text { Mean } \\
\text { cover of } \\
\text { tree } \\
\text { layer } \\
(\%)\end{array}$ & $\begin{array}{l}\text { Mean } \\
\text { number } \\
\text { of } \\
\text { woody } \\
\text { species }\end{array}$ \\
\hline IP & 11 & 1.12 & 357.51 & 0.48 & 0.05 & 0.00 & 0 \\
\hline PP & 17 & 0.40 & 350.73 & 0.81 & 0.26 & 0.00 & 1 \\
\hline ES & 20 & 0.68 & 256.82 & 0.29 & 0.71 & 0.04 & 2 \\
\hline $\mathrm{EF}$ & 8 & 1.89 & 1132.20 & 0.61 & 0.46 & 0.19 & 5 \\
\hline
\end{tabular}




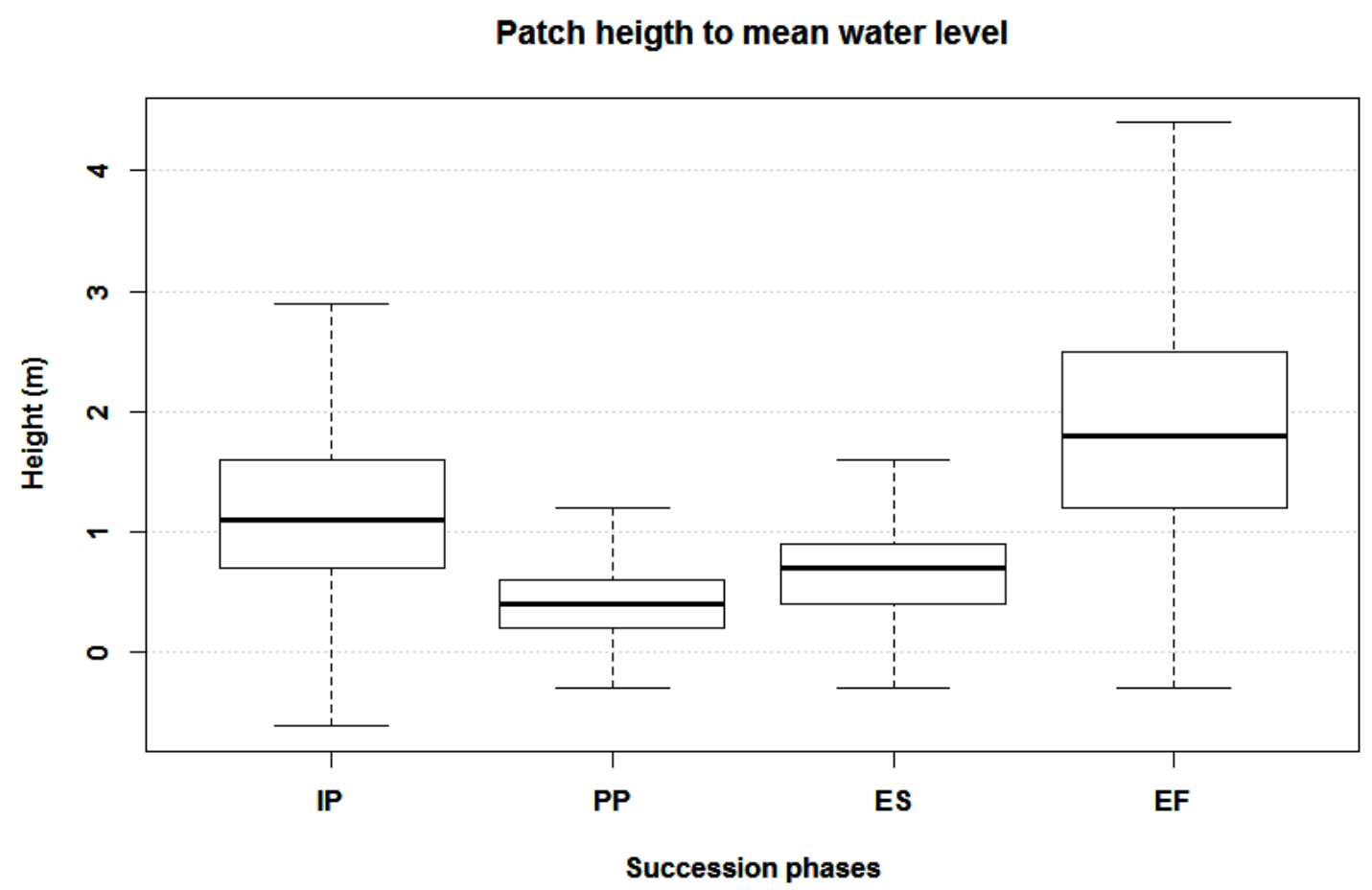

Figure S1. Patch height to mean water level grouped by succession phase (IP - Initial phase, PP - Pioneer phase, ES - Early succession woodland phase and EF - Established forest phase). 


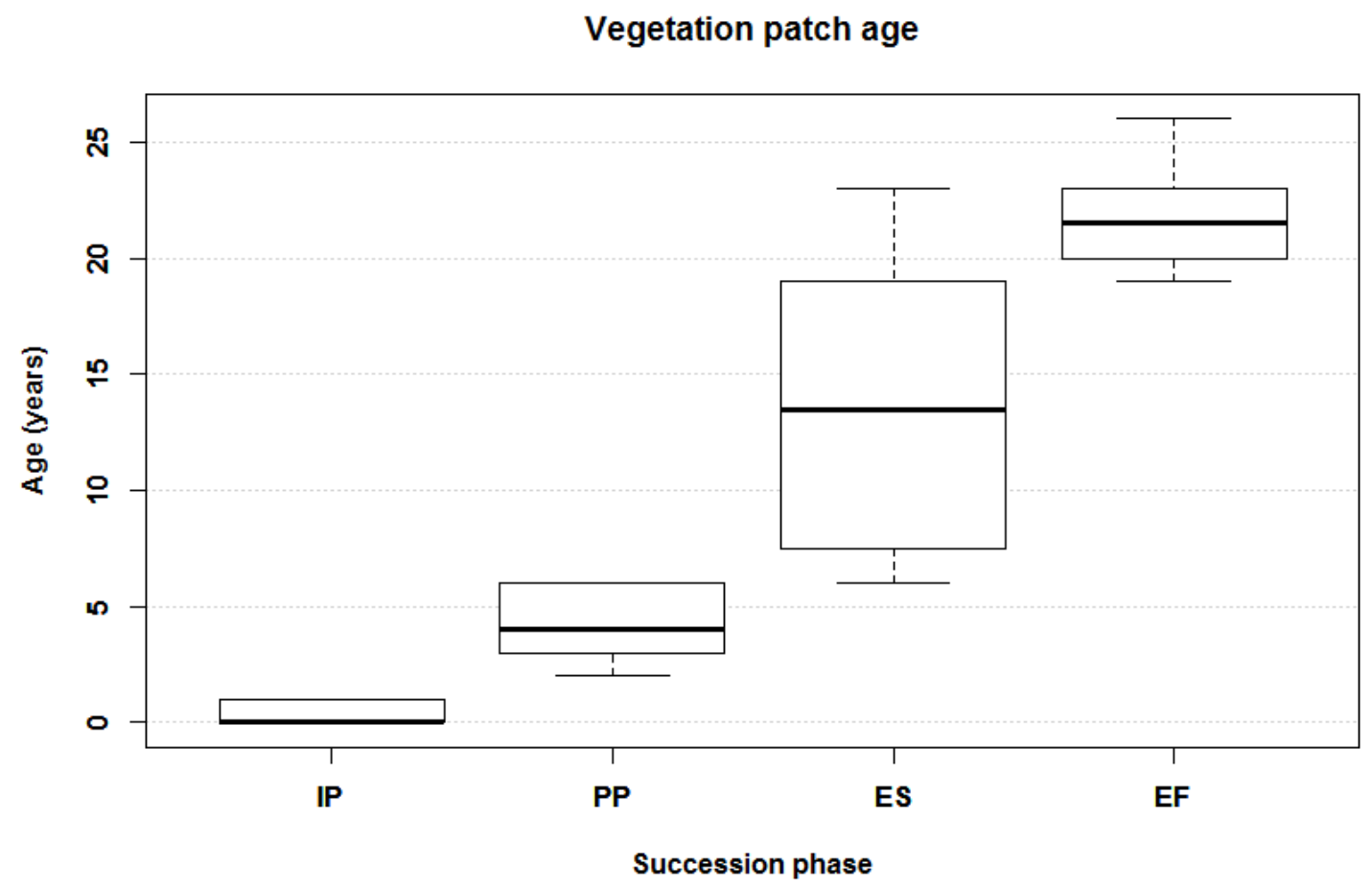

Figure S2. Patch age grouped by succession phase (IP - Initial phase, PP - Pioneer phase, ES - Early succession woodland phase and EF - Established forest phase).

Table S6. Number of captured cyprinid individuals throughout different sampling seasons in Ocreza river basin.

\begin{tabular}{llllll}
\hline Common name & Cyprinid Species & Spring & Summer & Autumn & Total \\
\hline Iberian barbel & Luciobarbus bocagei & 666 & 300 & 102 & 1068 \\
$\begin{array}{l}\text { Iberian straight } \\
\text { mouth-nase }\end{array}$ & $\begin{array}{l}\text { Pseudochondrostoma } \\
\text { polylepis }\end{array}$ & 46 & 62 & 102 & 210 \\
Calandino & Squalius alburnoides & 277 & 364 & 134 & 775 \\
Southern Iberian chub & Squalius pyrenaicus & 9 & 0 & 29 & 38 \\
\hline & Total & 998 & 726 & 367 & 2091 \\
\cline { 2 - 5 }
\end{tabular}



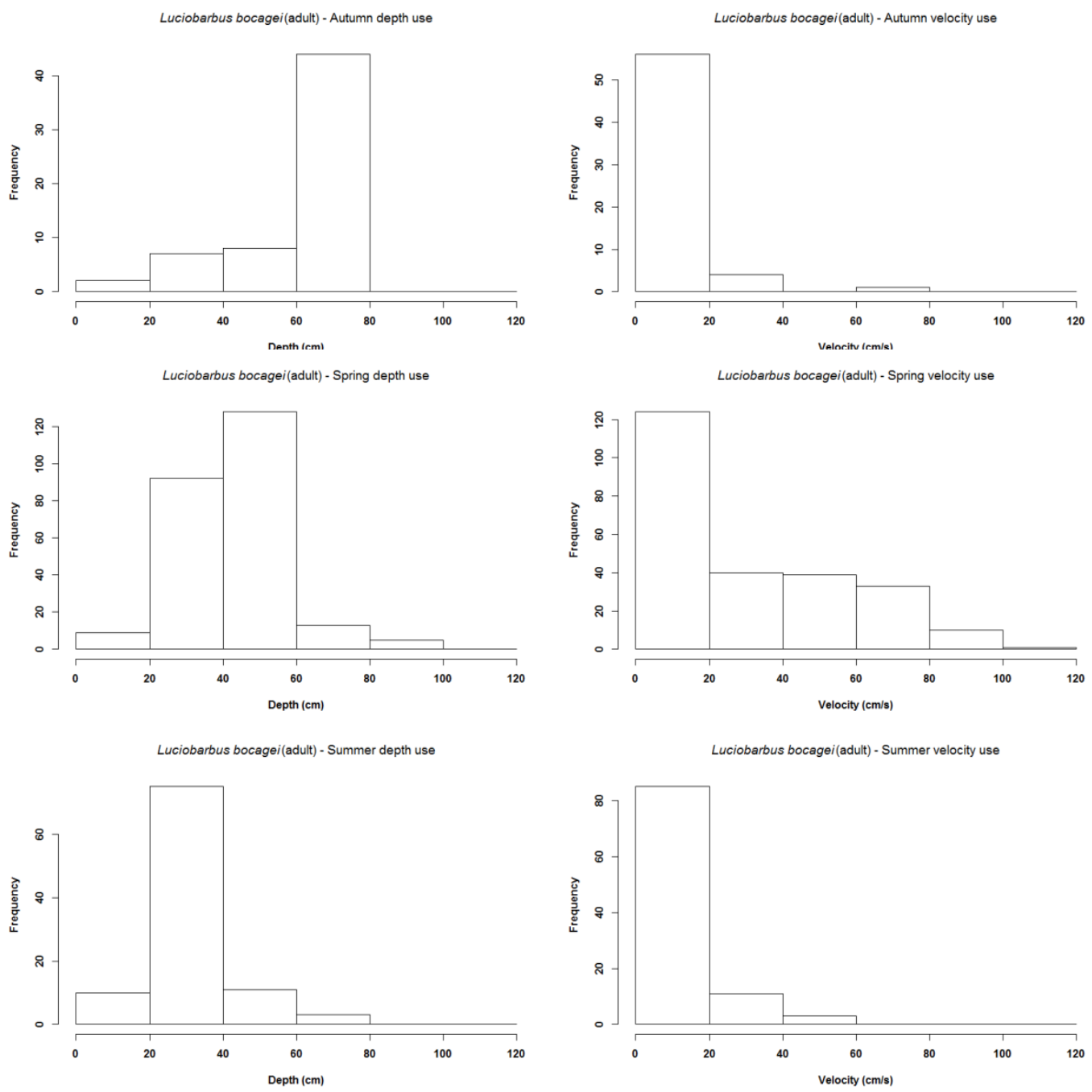

Figure S3. Use frequency of Luciobarbus bocagei adults for water depth (cm) and flow velocity $(\mathrm{cm} / \mathrm{s})$ during Autumn, Spring and Summer. 

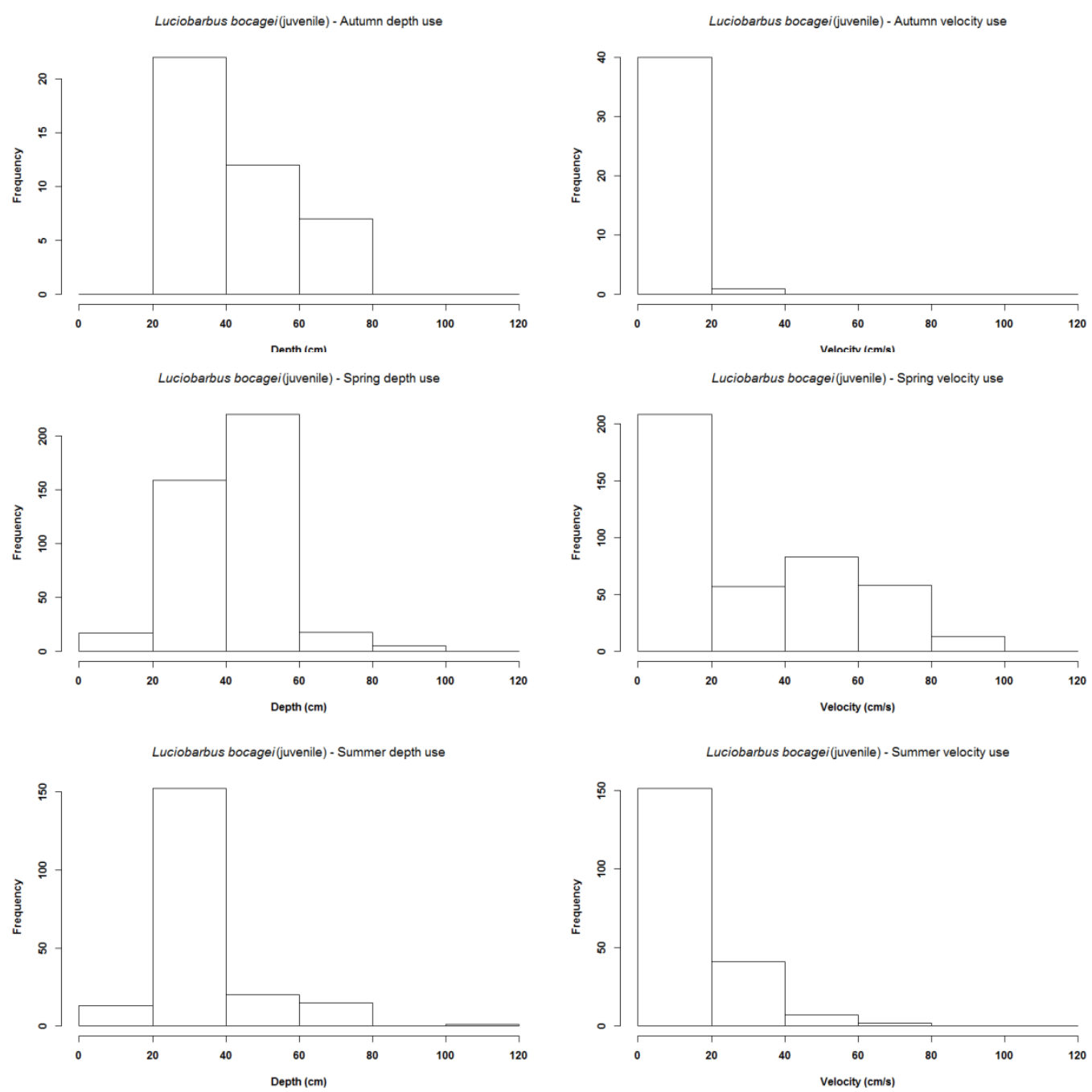

Figure S4. Use frequency of Luciobarbus bocagei juveniles for water depth $(\mathrm{cm})$ and flow velocity $(\mathrm{cm} / \mathrm{s})$ during Autumn, Spring and Summer. 

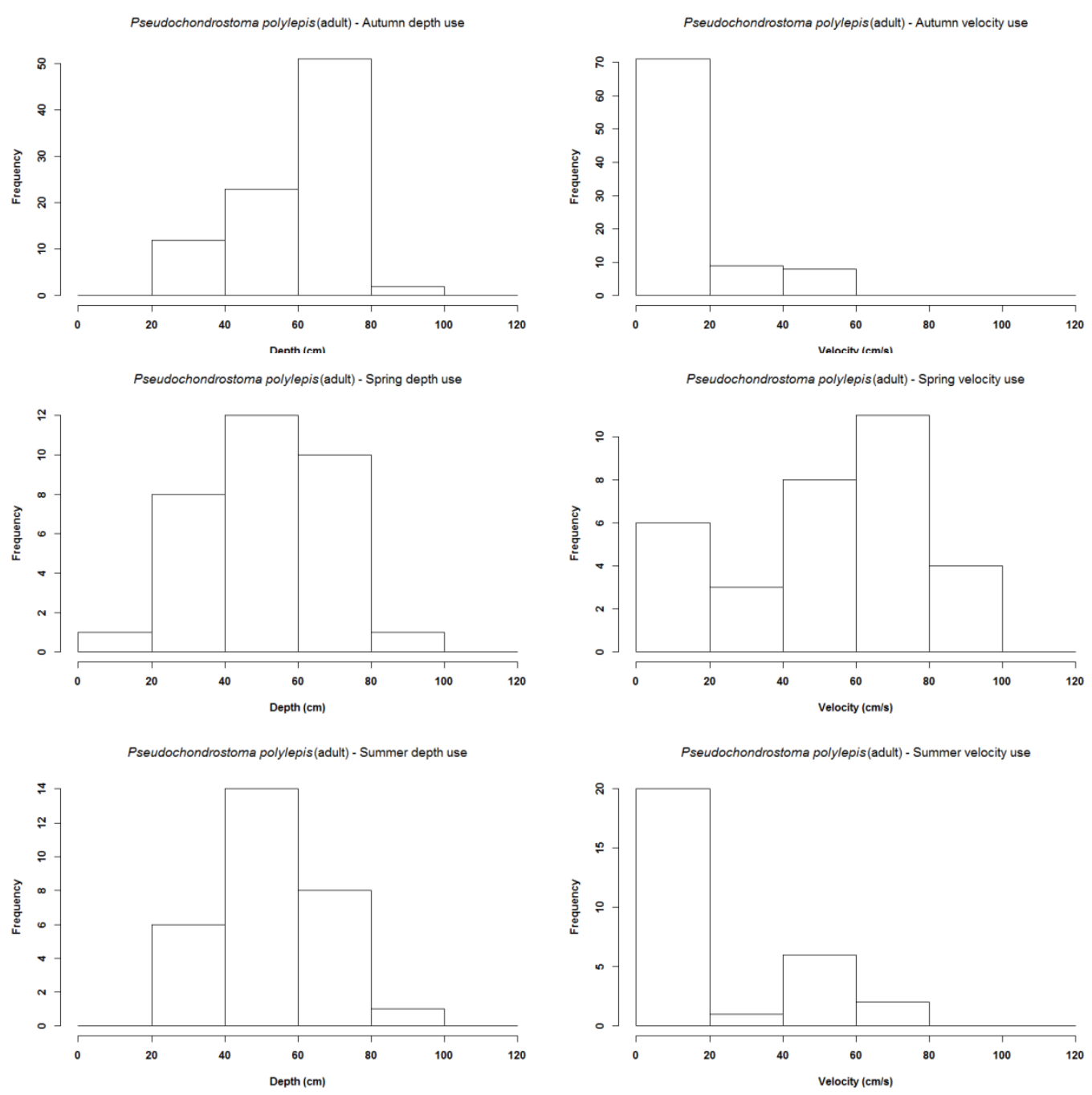

Figure S5. Use frequency of Pseudochondrostoma polylepis adults for water depth $(\mathrm{cm})$ and flow velocity $(\mathrm{cm} / \mathrm{s})$ during Autumn, Spring and Summer. 

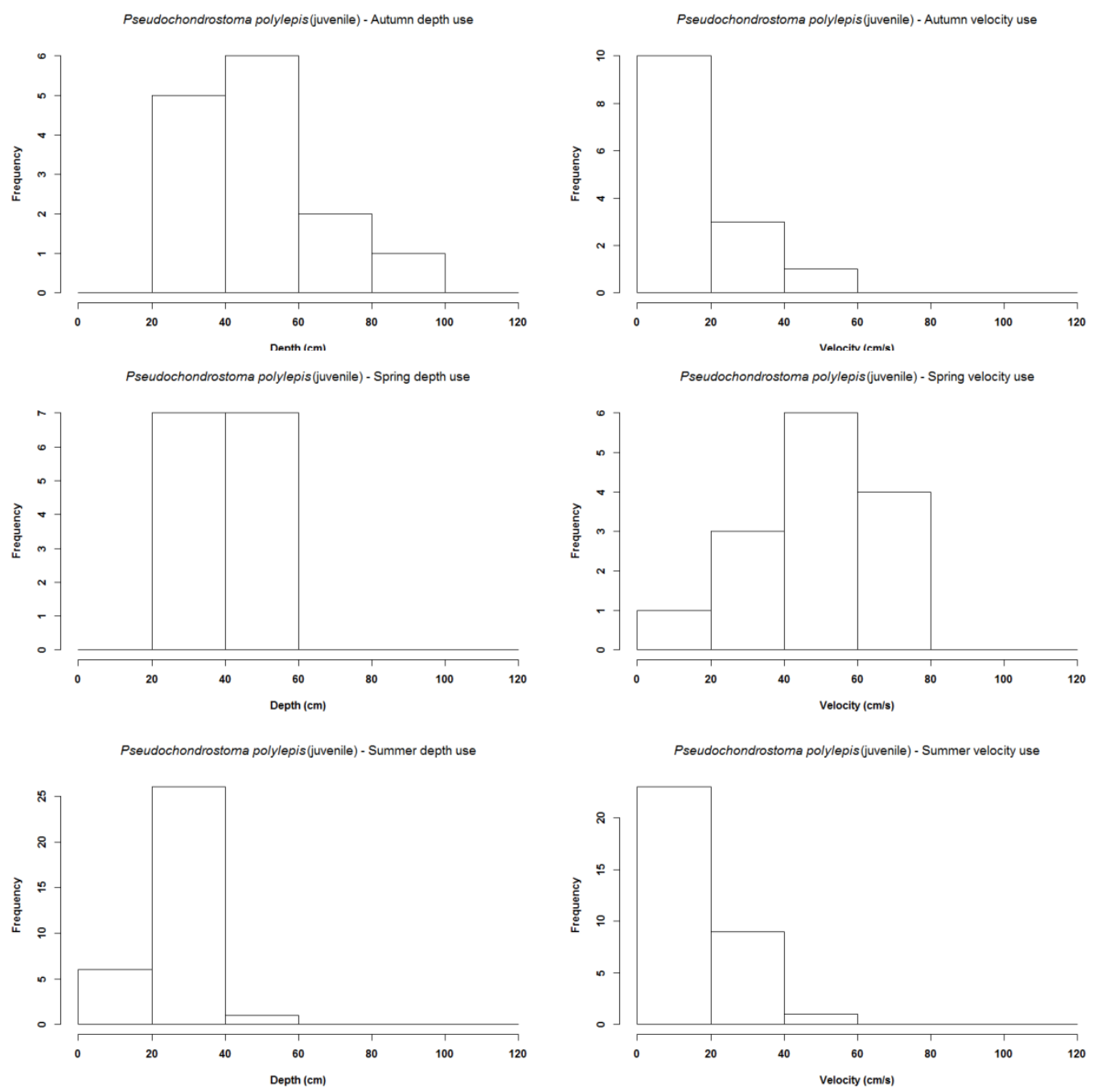

Figure S6. Use frequency of Pseudochondrostoma polylepis juveniles for water depth $(\mathrm{cm})$ and flow velocity $(\mathrm{cm} / \mathrm{s})$ during Autumn, Spring and Summer. 

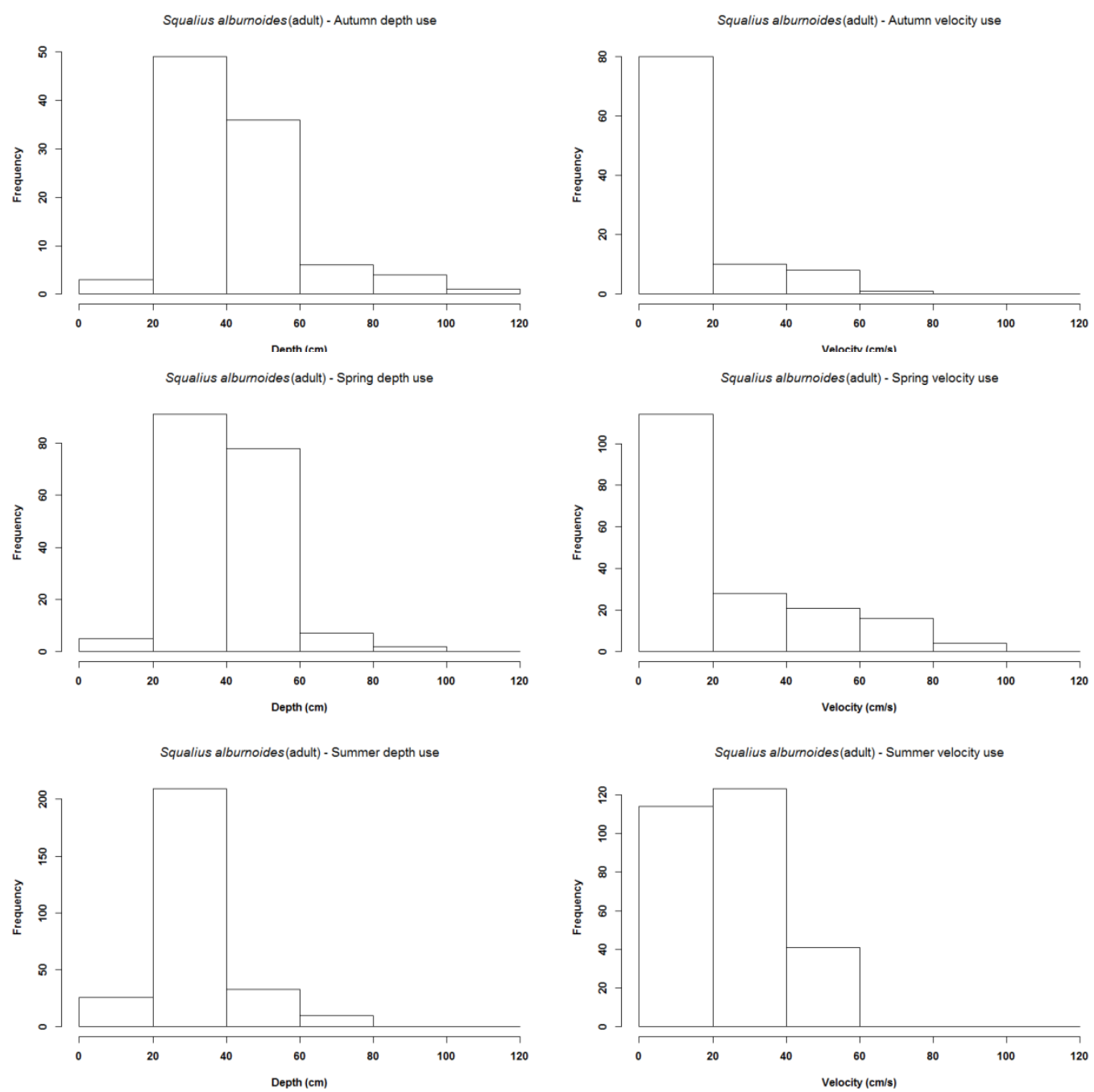

Figure S7. Use frequency of Squalius alburnoides adults for water depth (cm) and flow velocity $(\mathrm{cm} / \mathrm{s})$ during Autumn, Spring and Summer. 

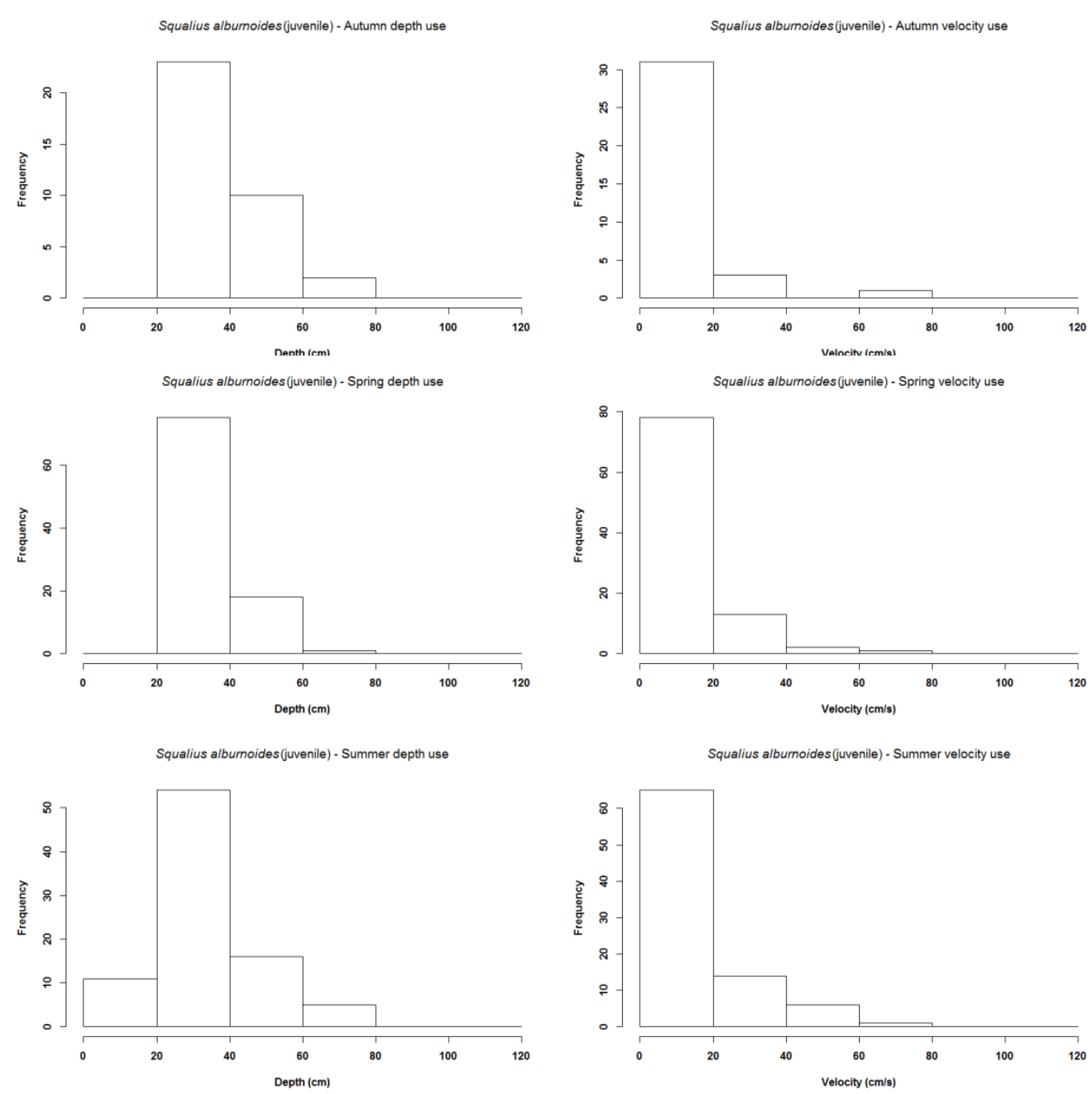

Figure S8. Use frequency of Squalius alburnoides juveniles for water depth $(\mathrm{cm})$ and flow velocity $(\mathrm{cm} / \mathrm{s})$ during Autumn, Spring and Summer. 

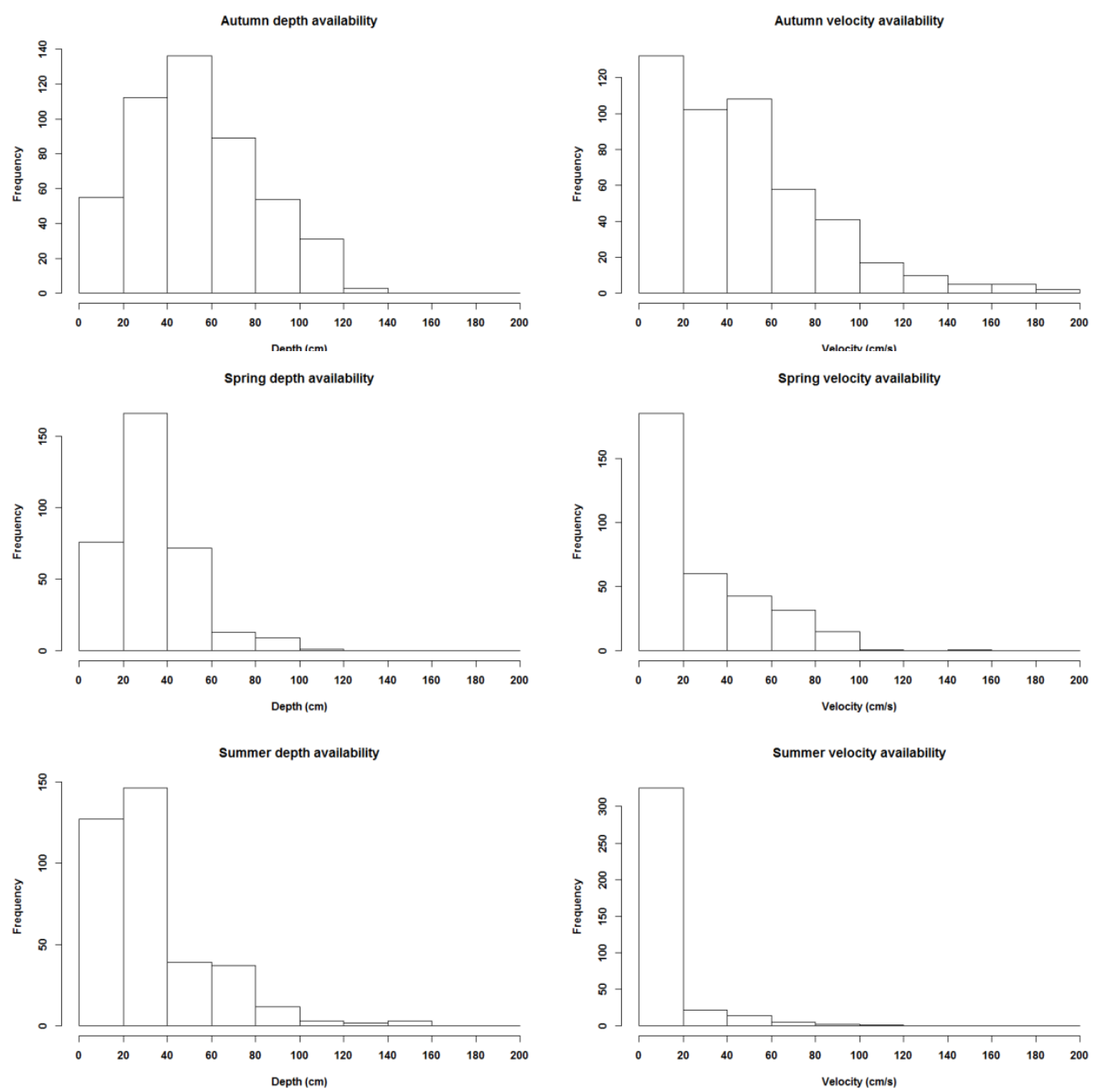

Figure S9. Habitat availability for water depth $(\mathrm{cm})$ and flow velocity $(\mathrm{cm} / \mathrm{s})$ during Autumn, Spring and Summer. 

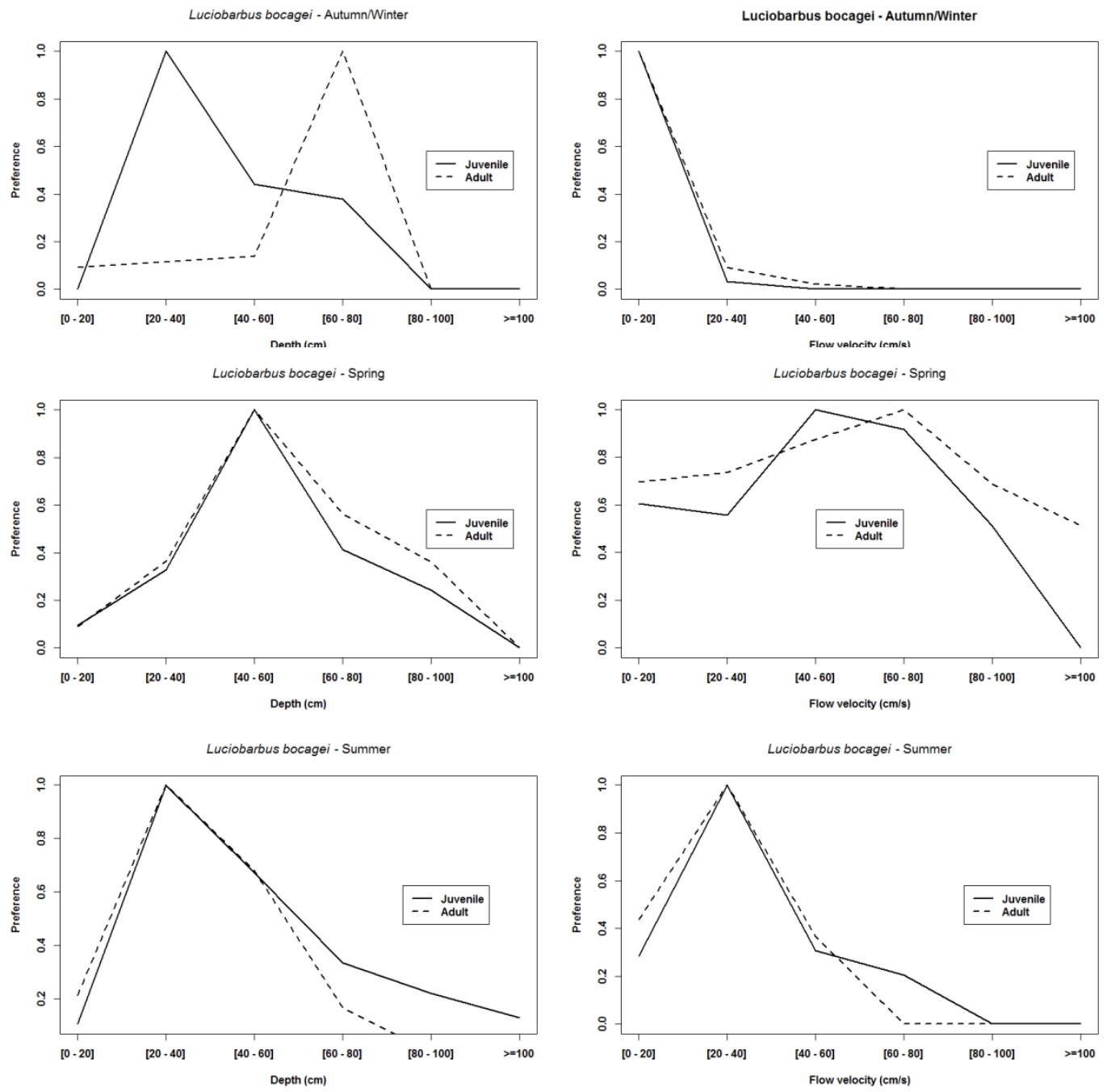

Figure S10. Habitat preference of Luciobarbus bocagei for water depth (cm) and flow velocity $(\mathrm{cm} / \mathrm{s})$ during Autumn, Spring and Summer. 

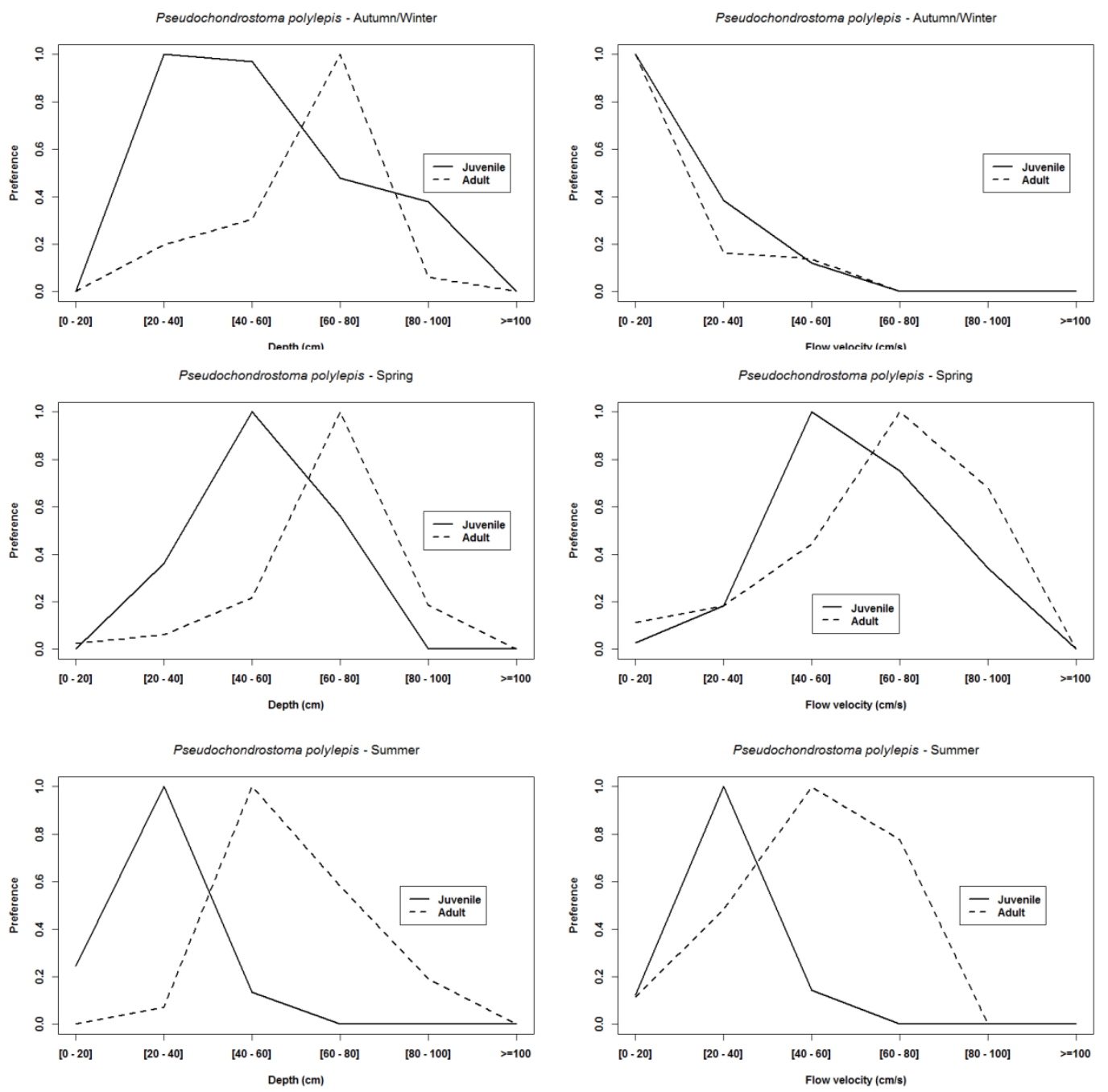

Figure S11. Habitat preference of Pseudochondrostoma polylepis for water depth $(\mathrm{cm})$ and flow velocity $(\mathrm{cm} / \mathrm{s})$ during Autumn, Spring and Summer. 

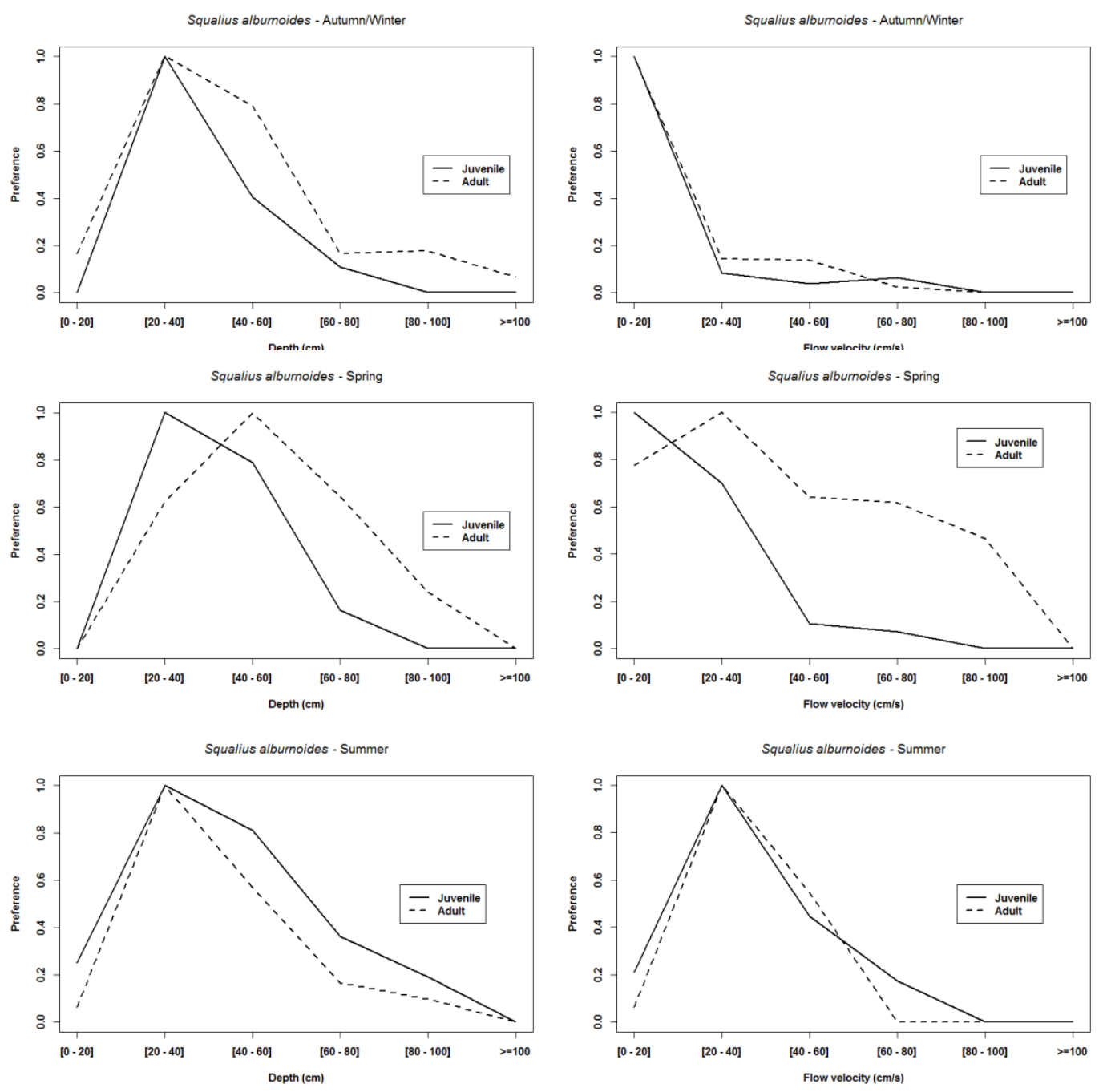

Figure S12. Habitat preference of Squalius alburnoides for water depth (cm) and flow velocity $(\mathrm{cm} / \mathrm{s})$ during Autumn, Spring and Summer. 
Supplement S3: $x^{2}$ test $p$-values for the equality of proportions between the Natural habitat and the Eflow and Eflow\&flush habitats

Table S7. Equality of proportions between Natural habitat and Eflow habitat (H0: WUA's have the same true proportion). Species codes stand for Lb - Luciobarbus bocagei; $\mathrm{Pp}$ - Pseudochondrostoma polylepis; $\mathrm{Sa}$ - Squalius alburnoides.

\begin{tabular}{lllllll}
\hline Month & Lb_juv & Lb_adult & Pp_juv & Pp_adult & Sa_juv & Sa_adult \\
\hline Oct & 0.000216 & 0.582952 & $6.21 \mathrm{E}-05$ & 0.940216 & 0.323139 & 0.297538 \\
Nov & 0.000148 & 0.530769 & $1.74 \mathrm{E}-05$ & 0.896214 & 0.370834 & 0.329465 \\
Dec & 0.000608 & 0.244612 & $1.17 \mathrm{E}-07$ & 0.483554 & 0.668862 & 0.868717 \\
Jan & 0.000519 & 0.257775 & $1.41 \mathrm{E}-07$ & 0.510652 & 0.789124 & 0.991580 \\
Feb & 0.000229 & 0.388867 & $1.60 \mathrm{E}-06$ & 0.723955 & 0.587666 & 0.438736 \\
Mar & 0.005353 & 0.053108 & 0.462443 & 0.688765 & 0.035578 & $7.11 \mathrm{E}-05$ \\
Apr & 0.005855 & 0.037780 & 0.398626 & 0.717639 & 0.041171 & $7.97 \mathrm{E}-05$ \\
May & 0.001723 & 0.709184 & 0.524064 & 0.379709 & 0.005987 & $1.65 \mathrm{E}-05$ \\
Jun & 0.795967 & 0.142917 & 0.610609 & 0.548629 & 0.248239 & 0.823737 \\
Jul & 0.878494 & 0.902296 & 0.931916 & 0.887568 & 0.978652 & 0.745271 \\
Aug & 0.878494 & 0.902296 & 0.931916 & 0.887568 & 0.978652 & 0.745271 \\
Sep & 0.308822 & 0.576689 & 0.272860 & 0.683524 & 0.562069 & 0.474081 \\
\hline
\end{tabular}


Table S8. Equality of proportions between Natural habitat and Eflow\&flush habitat (H0: WUA's have the same true proportion). Species codes stand for Lb - Luciobarbus bocagei; $\mathrm{Pp}$ - Pseudochondrostoma polylepis; $\mathrm{Sa}$ - Squalius alburnoides.

\begin{tabular}{lllllll}
\hline Month & Lb_juv & Lb_adult & Pp_juv & Pp_adult & Sa_juv & Sa_adult \\
\hline Oct & 0.850180 & 0.975380 & 0.937319 & 0.982124 & 0.889660 & 0.929312 \\
Nov & 0.837909 & 0.975984 & 0.918213 & 0.986510 & 0.879203 & 0.920722 \\
Dec & 0.088235 & 0.361823 & 0.101082 & 0.422564 & 0.247659 & 0.189515 \\
Jan & 0.146970 & 0.462011 & 0.162449 & 0.516233 & 0.312924 & 0.256096 \\
Feb & 0.721348 & 0.970973 & 0.770267 & 0.981017 & 0.757865 & 0.774812 \\
Mar & 0.934309 & 0.853870 & 0.863878 & 0.997663 & 0.934126 & 0.976693 \\
Apr & 0.929003 & 0.844001 & 0.858171 & 0.998189 & 0.928228 & 0.970043 \\
May & 0.984099 & 0.955728 & 0.944510 & 0.987440 & 0.993220 & 0.956985 \\
Jun & 0.951283 & 0.919954 & 0.985273 & 0.981322 & 0.998841 & 0.911286 \\
Jul & 0.891434 & 0.870963 & 0.924438 & 0.982402 & 0.891084 & 0.850580 \\
Aug & 0.891434 & 0.870963 & 0.924438 & 0.982402 & 0.891084 & 0.850580 \\
Sep & 0.747660 & 0.865492 & 0.690731 & 0.875758 & 0.796120 & 0.742462 \\
\hline
\end{tabular}

